\title{
Avaliação do efeito de deformação plástica na dureza, microestrutura e propriedades magnéticas de um aço inoxidável AISI 316L
}

\author{
Evaluation of the effect of plastic \\ deformation on the microstructure, \\ hardness and magnetic properties \\ of AISI type 316L stainless steel
}

\author{
Allan Victor Mathias Marques ${ }^{1}$, Kerciely Martins do Carmo ${ }^{1}$, \\ Wivyan Castro Lage ${ }^{1}$, Ricardo Luiz Perez Teixeira ${ }^{2}$, \\ José Carlos de Lacerda ${ }^{2}$, Cynthia Helena Soares Bouças Teixeira ${ }^{2}$, \\ Ricardo Shitsuka ${ }^{1}$
}

\footnotetext{
${ }^{1}$ Universidade Federal de Itajubá - Campus Itabira, 35900-000, Itabira, MG, Brasil. e-mail: allanvmmarques@hotmail.com, kercielymartins@unifei.edu.br,wivyan.cl@hotmail.com,rshitsuka@uol.com.br

${ }^{2}$ Grupo de Pesquisa em Sistemas de Exaustão da UNIFEI, 35900-000, Itabira, MG, Brasil.

e-mail: ricardo.luiz@unifei.edu.br, jlacerda@unifei.edu.br, cyrilet@gmail.com
}

\section{RESUMO}

O aço AISI 316L é muito aplicado como biomaterial de baixo custo em próteses e equipamentos cirúrgicos, dentre outras aplicações. Estas aplicações se devem à sua boa resistência à corrosão em presença de meios contendo fluidos corporais. Muito embora o aço apresente as características mencionadas, estas poderão ser comprometidas com a formação de martensita alfa linha (fase magnética) induzida por deformação a frio. Neste caso, o presente trabalho, teve como objetivo investigar os efeitos de laminação a frio no surgimento de fase martensítica. Para tanto foram realizadas três diferentes deformações. A chapa como recebida e as amostras deformadas foram submetidas a ensaios de ferritoscopia, microdureza Vickers e análise microestrutural por microscopia eletrônica de varredura, MEV. Foi observado que nas amostras deformadas a frio houve o surgimento de fase magnética (provavelmente martensita alfa linha) e aumento da microdureza, devido ao encruamento e à presença de fases induzidas por deformação.

Palavras-chave: AISI 316L; Martensita induzida por deformação; Fase magnética; Laminação a frio.

\section{ABSTRACT}

AISI 316L steel is widely used as a low cost biomaterial in prostheses and surgical equipment, among other applications. These applications are due to their good corrosion resistance in the presence of environment containing body fluids. Although this steel has the mentioned characteristics, it may be compromised with the formation of alpha prime martensite (magnetic phase) induced by plastic deformation. In this case, the present work aimed to investigate the cold rolling effects on the martensitic phase formation. For that, three different deformations were performed. The plate as received and the deformed samples were subjected to ferritoscopy, Vickers microhardness and microstructural analysis by scanning electron microscopy, SEM. It was observed that in cold deformed samples there was the magnetic phase appearance (probably alpha prime martensite) and microhardness increase, due to the hardening and the deformation induced phases presence.

Keywords: AISI 316L; Martensite induced by deformation; Magnetic phase, Cold rolling.

\section{INTRODUÇÃO}

Os aços inoxidáveis da série AISI 3XX, baixo carbono, são materiais bastante utilizados na confecção de equipamentos cirúrgicos, próteses de baixo custo dentre outras aplicações. A característica de biocompatibi- 
lidade atribuída aos aços AISI 3XX, baixo carbono, está relacionada com sua condição de predominância total de microestrutura austenítica. Qualquer alteração nesta condição, como por exemplo, o surgimento de uma nova fase ou de algum precipitado pode comprometer a sua utilização como biomaterial [1-4]. Especialmente o aço AISI 316L, se destaca dentre os demais aços da série 3XX pela sua maior resistência à corrosão em meios ácidos. Esta característica pode ser atribuída à presença de cromo, níquel e molibdênio na sua composição. Há que ressaltar ainda a sua excelente soldabilidade motivada pelo seu baixo teor de carbono (máximo 0,08\% em massa). O aço AISI 316L também apresenta boas propriedades mecânicas, alta tenacidade e boa trabalhabilidade. O conjunto de características mencionadas são essenciais para a seleção de um biomaterial metálico em certas aplicações, como por exemplo, em próteses de tecido duro [2-6].

Segundo a norma ISO 5832-1 [4], a microestrutura de aços AISI 3XX destinados a aplicações como biomateriais devem ser constituídos apenas pela fase austenítica. De acordo com a Norma citada, a presença de ferrita identificada por ampliação de 100x no microscópio desqualifica o aço como biomaterial. Ainda, o aço não deve ter a presença de inclusões não metálicas (sulfetos, aluminatos, silicatos e óxidos globulares). Estas inclusões podem aumentar a susceptibilidade à ocorrência de corrosão localizada [7, 8].

O aço AISI 316L, assim como os demais aços inoxidáveis austeníticos, apresentam uma microestrutura metaestável. Neste caso, os referidos aços podem se transformar em martensita alfa linha ( $\alpha$ ') que é ferromagnética. Esta fase pode ocorrer por deformação plástica. Este tipo de transformação é denominado efeito TRIP (Transformation Induced Plasticity). Dessa forma, o efeito TRIP pode ocorrer em processos de conformação mecânica a frio. A martensita é formada a partir da austenítica devido ao efeito energético de empilhamento. A fase martensítica induzida altera as características mecânicas do aço AISI 316L elevando seu limite de resistência e sua dureza. $\mathrm{O}$ aumento de limite de resistência e dureza ocorre tanto pela presença da martensita como pelo encruamento produzido pela conformação a frio. O surgimento da martensita diminui a resistência à corrosão e torna o aço ferromagnético. Essas características são comprometedoras para aplicações do aço como biomaterial metálico [9-19].

Conforme o exposto, o presente artigo se apresenta com o objetivo de analisar o efeito TRIP produzido por laminação a frio AISI tipo 316L.

\section{MATERIAIS E MÉTODOS}

Na Tabela 1 apresenta-se a composição química do aço AISI 316L, conforme fornecido pelo fabricante.

Tabela 1: composição química do aço AISI 316L (porcentagem em massa).

\begin{tabular}{|c|c|c|c|c|c|c|c|c|}
\hline $\mathrm{C}$ & $\mathrm{Cr}$ & $\mathrm{Ni}$ & $\mathrm{Mo}$ & $\mathrm{Cu}$ & $\mathrm{Si}$ & $\mathrm{P}$ & $\mathrm{S}$ & $\mathrm{Fe}$ \\
\hline 0,03 & 17,5 & 12,3 & 2,5 & 0,01 & 0,6 & 0,02 & 0,005 & Balanço \\
\hline
\end{tabular}

\subsection{Laminação a frio}

Chapas de aço AISI 316L laminadas a frio com $2 \mathrm{~mm}$ de espessura foram usinadas por eletroerosão e produzidas 4 amostras nas dimensões $25 \mathrm{~mm} \times 10 \mathrm{~mm}$ [20, 21]. As amostras foram laminadas a frio. Para tanto foi utilizado um laminador de laboratório, conforme apresentado na Figura 1.

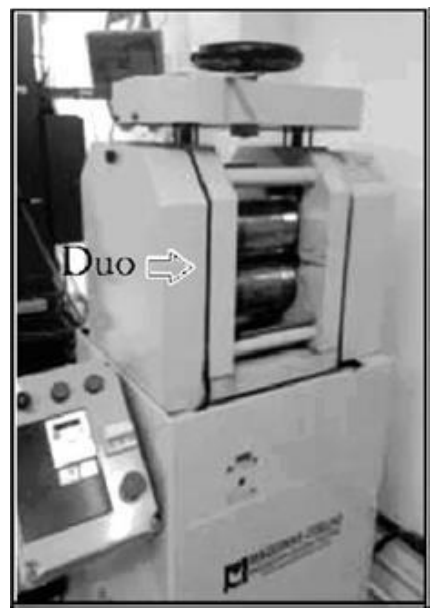

Figura 1: laminador de laboratório. 


\subsection{Ferritoscopia}

A morfologia da austenita, a composição química, o estado de tensões durante a deformação e a temperatura são fatores influenciadores na transformação martensítica [2]. A transformação gradual da austenita para martensita induzida por deformação associado com o inevitável encruamento, aumentam a dureza do aço. A martensita normalmente tem característica ferromagnética. A técnica da ferritoscopia pode ser empregada para a detecção da presença de fases magnéticas na microestrutura de aços [22-24]. Assim, foi empregado um ferritoscópio modelo de Fischer MP3C. Foram realizadas 10 medições em cada uma das amostras com as seguintes deformações por laminação a frio: $0 \%$ (chapa como recebida), 30\%, 50\% e 70\% de redução na espessura. Na Figura 2 são apresentadas as amostras cujas espessuras finais foram de aproximadamente 2,0 $\mathrm{mm}, 1,4 \mathrm{~mm}, 1,0 \mathrm{~mm}$ e $0,6 \mathrm{~mm}$.

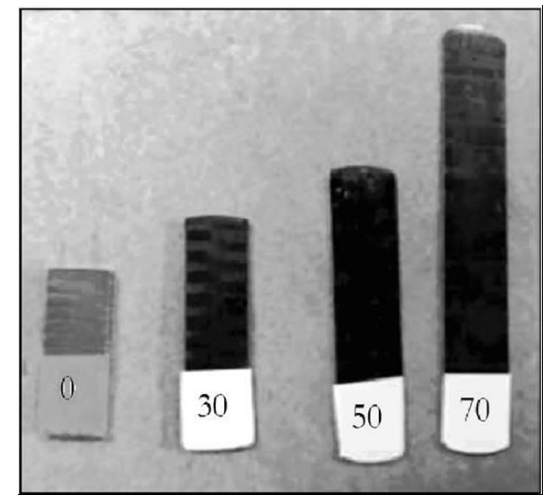

Figura 2: Amostras com respectivas deformações.

\subsection{Metalografia}

As amostras foram preparadas para análises metalográficas visando o estudo de suas microestruturas nas condições de deformação de $0 \%, 30 \%, 50 \%$ e $70 \%$ de redução nas espessuras. Para tanto, as amostras foram cortadas utilizando-se uma máquina de corte com disco abrasivo diamantado de baixa rotação. Os cortes foram realizados sob refrigeração a água.

Na sequência, as amostras cortadas foram submetidas à lixamento (granulometrias: 320\#, 400\#, 640\#, P800, P1200). Após essa etapa, foi realizado o polimento. Em ambos os casos, lixamento e polimento, foi utilizada uma politriz rotativa marca AROTEC. No caso do polimento, foi utilizado disco de feltro embebido em solução de alumina de $3 \mu \mathrm{m}$ e pasta de diamante de $1 \mu \mathrm{m}$ na última etapa do polimento.

$\mathrm{O}$ ataque das amostras foi realizado utilizando-se solução Murakami (10 g de ferrocianeto de potássio e $10 \mathrm{~g}$ de hidróxido de sódio solubilizados em $100 \mathrm{ml}$ de água). Este tipo de ataque é próprio para a revelação de martensita $\alpha$ ' em aços inoxidáveis [24, 25].

Foram obtidas imagens da microestrutura das amostras atacadas utilizando-se tanto de microscopia óptica (Figura 5) quando de microscopia eletrônica de varredura, MEV (Figura 6). Para a obtenção das imagens foram utilizados, respectivamente, um microscópio óptico metalográfico marca OPTON e um MEV TESCAN VEGA3. Foi utilizado também o recurso de espectroscopia por energia dispersiva (EDS), marca BRUKER, acoplado ao MEV, visando detectar as composições químicas presentes na superfície.

\subsection{Microdureza Vickers}

As medições de microdurezas Vickers foram realizadas de acordo com a norma ASTM E 92:17 [26]. Para as medições da microdureza foi utilizado um microdurômetro WILSON modelo 402MVD, com carga de 100 gf por $10 \mathrm{~s}$. Foram realizadas 4 medidas em cada amostra. 


\section{RESULTADOS E DISCUSSÃO}

\subsection{Ferritoscopia}

Por ferritoscopia constatou-se elevação da permeabilidade magnética com a redução de espessura das amostras. Este comportamento pode ser atribuído à formação de martensita $\alpha^{\prime}$ induzida por conformação pela laminação a frio. Conforme a Figura 2, não foi detectada propriedade magnética na amostra do aço AISI 316L como recebida ( $0 \%$ de deformação). Com isso ficou confirmada a existência de microestrutura totalmente austenítica. Por outro lado, nas amostras que foram laminadas a frio houve elevação das propriedades magnéticas com o aumento da deformação. Isso pode ser atribuído ao surgimento de martensita $\alpha$ ' induzida pela laminação a frio, conforme Figura 3.

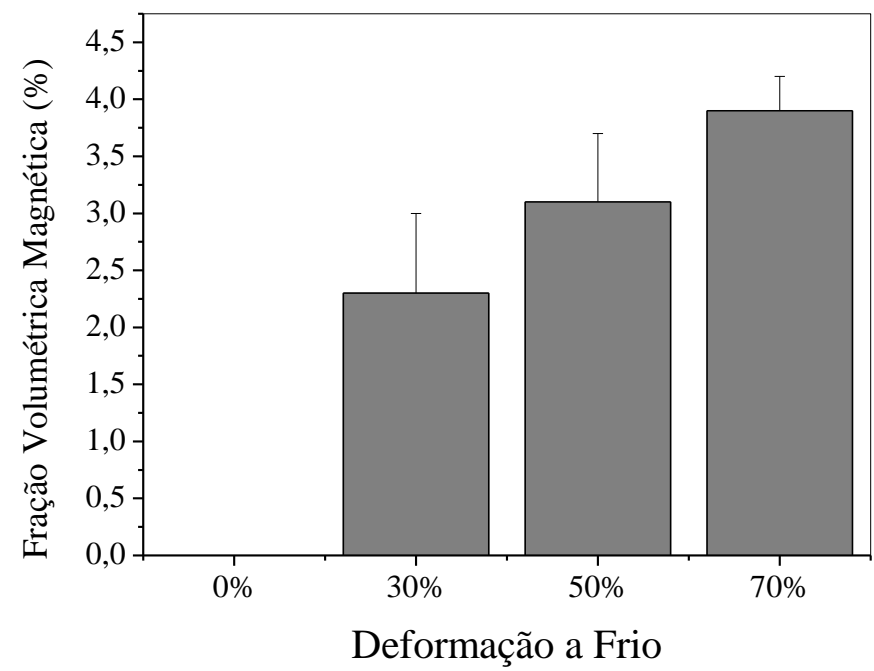

Figura 3: Fração magnética induzida pela deformação a frio.

A martensita $\alpha$ ' nucleia e cresce nas discordâncias existentes nas maclas produzidas por cisalhamento, assim como nas falhas de empilhamento (núcleos de formação de maclas). De acordo com a literatura [2729], a martensita $\alpha$ ' nucleia e cresce na forma de ripas na orientação [202] no plano (111) da austenita.

\subsection{Microdureza Vickers}

Na Figura 4 apresenta-se a evolução da microdureza Vickers motivada pela deformação por laminação a frio do aço AISI 316L. A microdureza cresceu com o aumento da deformação. Esse aumento ocorreu devido ao aumento gradual do encruamento associado à provável precipitação de martensita $\alpha^{\prime}$ [30,31]. 


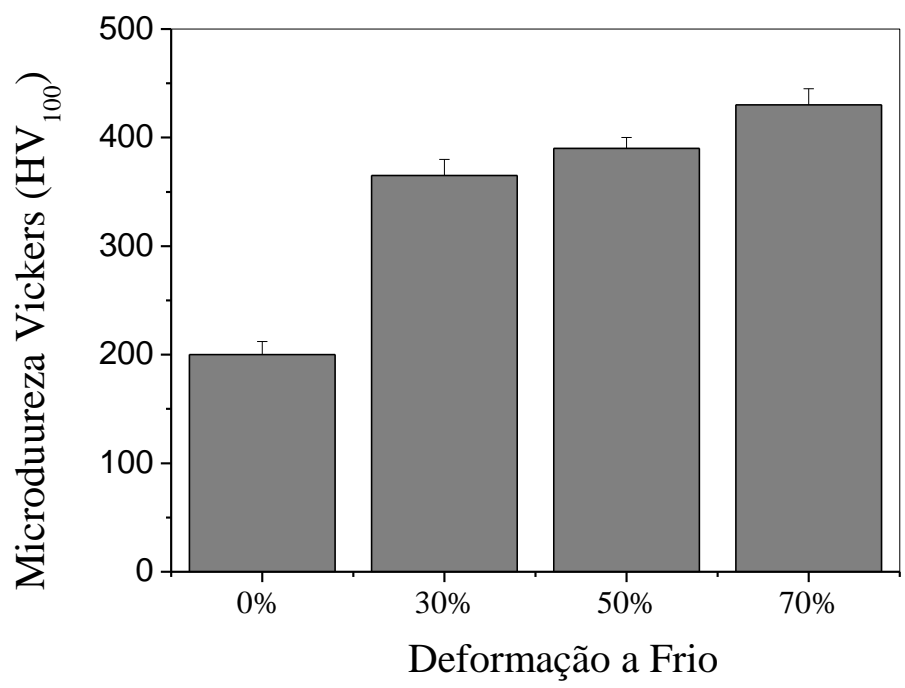

Figura 4: Variação de microdureza Vickers.

\subsection{Microscopia}

Na Figura 5 são apresentadas as micrografias do aço AISI 316L. Na Figura 5(a) aparecem destacadas por círculos tracejados a existência de grãos poligonais típicos da fase austenítica. Nas demais Figuras observamse grãos deformados no sentido da laminação, com destaque para a presença de maclas indicadas na Figura 5(b) [32]. 

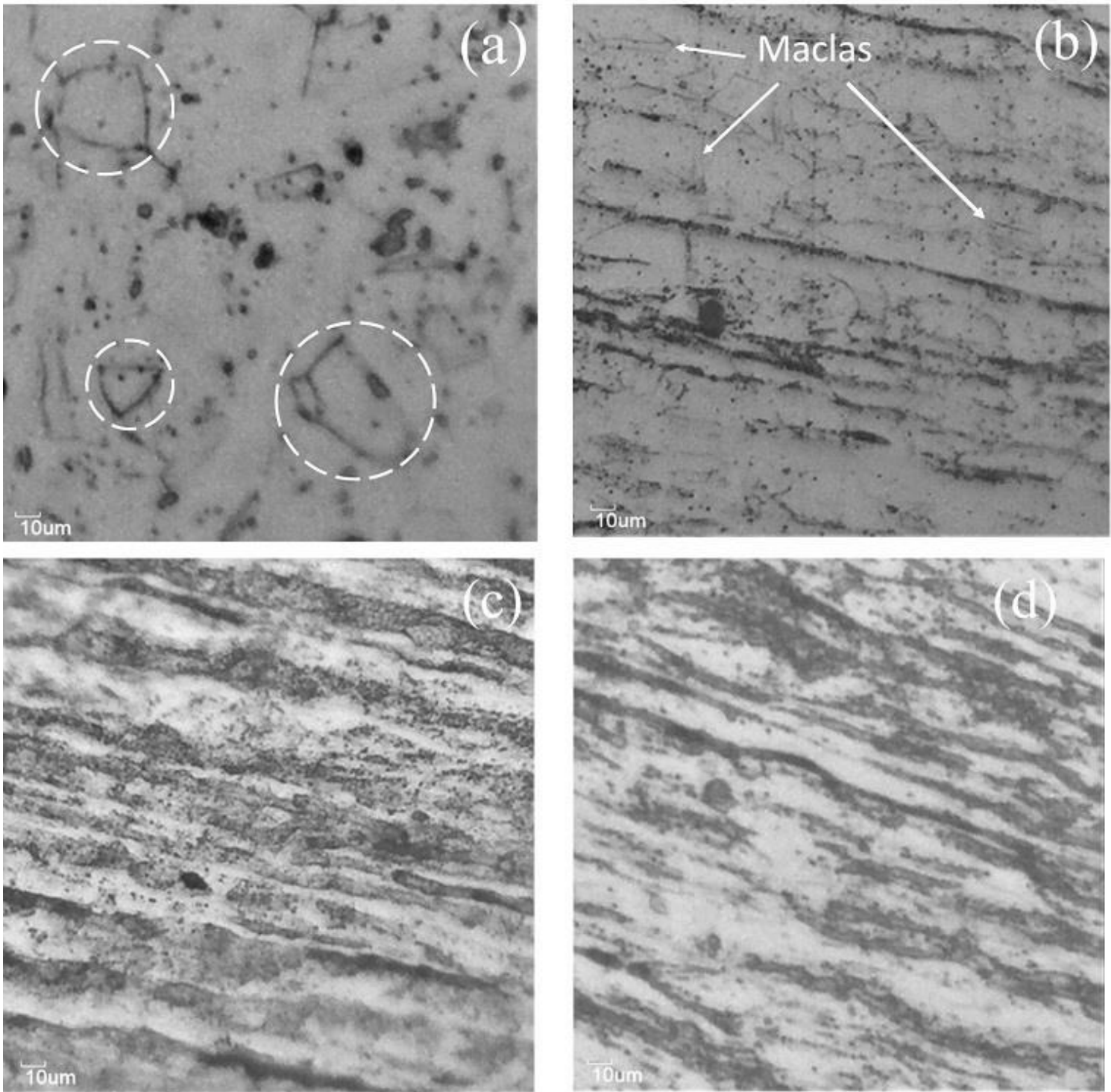

Figura 5: Micrografias ópticas do aço AISI 316L: (a) 0\% de deformação; (b) 30\% de deformação; (c) $50 \%$ de deformação; (d) $70 \%$ de deformação.

A deformação produzida pela laminação a frio, como mostrada na Figura $5(\mathrm{~b}, \mathrm{c}, \mathrm{d})$, favorece a formação de fase martensítica $\alpha^{\prime}$ induzida e, com isso, consequentemente originando fase magnética na liga. Essa fase magnética foi confirmada por ferritoscopia como mostrado na Figura 3. Vale ressaltar que a presença de fase magnética na liga pode comprometer a sua aplicação como biomaterial [4].

Na Figura 6 são apresentadas as micrografias do aço AISI 316L obtidas por MEV. Na Figura 6(a) a existência de grãos poligonais típicos da fase austenítica é confirmada. Também pode ser notada a existência de alguma maclas provavelmente originadas por eventual tratamento térmico ou deformação prévia do aço pelo fabricante considerando que a amostra em referência não foi laminada ( $0 \%$ de deformação). Na Figura 6(b), relativa a 30\% de deformação, são observadas maclas de deformação localizadas no interior de alguns grãos de austenita, conforme indicadas por setas [32]. Na Figura 6(c), relativa a 50\% de deformação, também pode ser observada a presença de algumas maclas no interior de grãos de austenita. Na Figura 6(d) observa-se o surgimento de algumas trincas e maclas deformadas devido provavelmente a elevada taxa de deformação (70\% de deformação). 

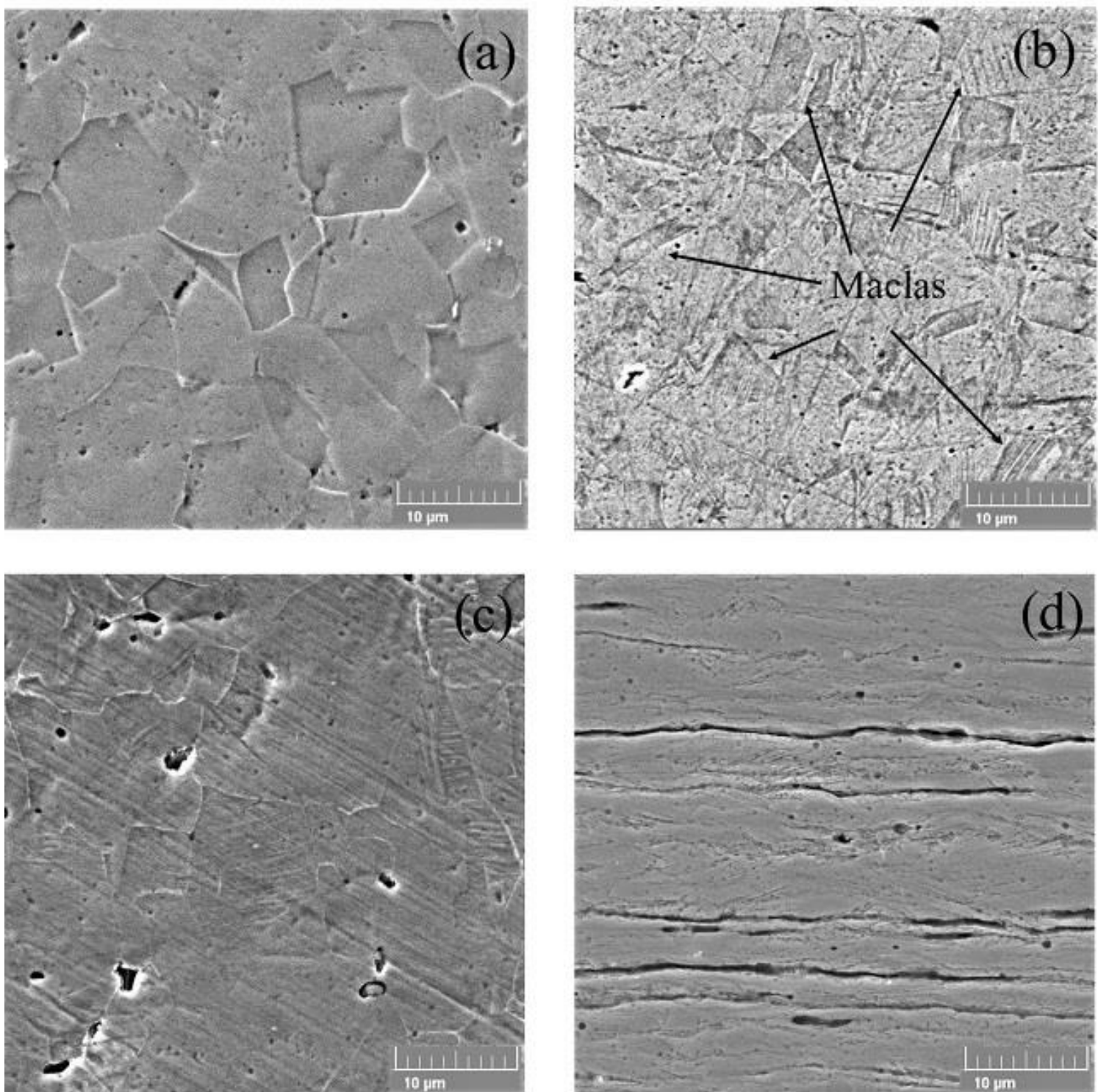

Figura 6: Micrografias por MEV do aço AISI 316L: (a) 0\% de deformação; (b) 30\% de deformação; (c) $50 \%$ de deformação; (d) $70 \%$ de deformação.

A presença de maclas de deformação nas amostras laminadas a frio é indicativo da formação de martensita $\alpha^{\prime}$. A martensita $\alpha^{\prime}$ tem tendência em nuclear heterogeneamente em regiões de alta energia com as maclas de deformação, contornos de grãos, subgrãos, bandas de cisalhamento e em falhas de empilhamento [33-36].

\section{CONCLUSÕES}

$>$ As amostras do aço AISI 316L laminadas a frio apresentaram aumento gradual da fração volumétrica magnética com o aumento do grau de deformação o que indica evidências do surgimento de martensita $\alpha$.

$>\mathrm{O}$ aumento da taxa de deformação por laminação a frio causou aumento gradual na dureza Vickers das amostras devido ao encruamento e à presença de fases induzidas por deformação. 
$>$ Observou-se o surgimento de maclas de deformação no interior dos grãos austeníticos nas amostras deformadas a $30 \%$ e $50 \%$.

$>\mathrm{Na}$ amostra deformada a $70 \%$ pode ser observada a presença de trincas e maclas deformadas no sentido da laminação.

\section{AGRADECIMENTOS}

Os autores agradecem à UNIFEI Itabira e ao GPESE pelo apoio a esta pesquisa.

\section{BIBLIOGRAFIA}

[1] MARIC, M., et al., "The Effect of Cold-Rolling on the Microstructure and Corrosion Behaviour of 316L Alloy in FLiNaK Molten Salt", Corrosion Science, 2018.

[2] ASTM F138-13a. Standard Specification for Wrought 18Chromium-14Nickel-2.5Molybdenum Stainless Steel Bar and Wire for Surgical Implants (UNS S31673), ASTM International, West Conshohocken, PA, 2013.

[3] ASTM F139-12. Standard Specification for Wrought 18Chromium-14Nickel-2.5Molybdenum Stainless Steel Sheet and Strip for Surgical Implants (UNS S31673), ASTM International, West Conshohocken, PA, 2012.

[4] ISO 5832-1. Implants for surgery - Metallic materials - Part 1: Wrought stainless steel, International Organization for Standardization, 2016.

[5] TEIXEIRA, R. L. P., GODOY, G. C. D., PEREIRA, M. M., "Calcium phosphate formation on alkalitreated titanium alloy and stainless steel", Materials Research. v. 7, n. 2, pp. 299-303, 2004.

[6] MINA, A., CAICEDO, H. H., UQUILLAS, J. A et al., "Biocompatibility behavior of b-tricalcium phosphate-chitosan coatings obtained on 316L stainless steel", Materials Chemistry and Physics. v. 175, pp. 6880, 2016.

[7] GRAHAM, C. D; LORENZ, B. E., "Delta ferrite is ubiquitous in type 304 stainless steel: Consequences for magnetic characterization", Journal of Magnetism and Magnetic Materials, v. 458, pp. 15-18, 2018.

[8] ZHENG, S., LI, C., QI, Y., et al., "Mechanism of (Mg,Al,Ca)-oxide inclusion-induced pitting corrosion in 316L stainless steel exposed to sulphur environments containing chloride ion", Corrosion Science, v. 67, p. 20-31, 2013.

[9] ZHANG, S. Y., COMPAGNON, E., GODIN, B. et al., "Investigation of martensite transformation in 316L stainless steel”, Materials Today: Proceedings S, v.2, pp. S251-S260, 2015.

[10] BIEHLER, J., HOCHE, H., OECHSNER, M.., "Nitriding behavior and corrosion properties of AISI 304L and 316L austenitic stainless steel with deformation-induced martensite", Surface and Coatings Technology. v. 324, pp. 121-128, 2017.

[11] MAUROTTO, A., TSIVOULAS, D., GU, Y., et al., "Effects of machining abuse on the surface properties of AISI 316L stainless steel", International Journal of Pressure Vessels and Piping, v. 151, pp. 35-44, 2017.

[12] MÜLLER-BOLLENHAGEN, C., ZIMMERMANN, M., CHRIST, H. J., "Very high cycle fatigue behaviour of austenitic stainless steel and the effect of strain-induced martensite", International Journal of $\mathrm{Fa}$ tigue. v. 32, pp. 936-942, 2010.

[13] SHRINIVAS, V., VARMA, S. K., MURR, L.E., "Deformation-Induced Martensitic Characteristics of 304 and 316 Stainless Steels During Room-Temperature Rolling", Met. Mater. Trans. A., v. 26A, pp. 661$671,1995$.

[14] SERRI, J., MARTINY, M., FERRON, G., "Finite element analysis of the effects of martensitic phase transformation in TRIP steel sheet forming", International Journal of Mechanical Sciences. v. 47, n. 6, p. 884-901, 2005.

[15] YEGANEH, A., OTOUKESH, B., KAGHAZIAN, P., et al, M. "Evaluation of the etiologies of implant fracture in patients with fractures of the implants of lower limbs' long bones", Medical Archives, v. 69. n. 6, pp. 405, 2015. 
[16] ONG, K. L., LOVALD, S., BLACK, J. Orthopaedic biomaterials in research and practice, CRC Press, 2014.

[17] BARBOSA, C., NASCIMENTO, J. L., CAMINHA, I. M. V., et al., "Premature failure in orthopedic implants: analysis of three different cases", Journal of failure analysis and prevention, v. 9, n. 1, pp. 67-73, 2009.

[18] TRIANTAFYLLIDIS, G. K., KAZANTZIS, A. V., KARAGEORGIOU, K. T., "Premature fracture of a stainless steel 316L orthopaedic plate implant by alternative episodes of fatigue and cleavage decoherence", Engineering Failure Analysis, v. 14, n. 7, pp. 1346-1350, 2007.

[19] AZEVEDO, C. R. F., HIPPERT Jr, E., "Failure analysis of surgical implants in Brazil", Engineering Failure Analysis, v. 9, n. 6, pp. 621-633, 2002.

[20] ASTM A240 / A240M-17, Standard Specification for Chromium and Chromium-Nickel Stainless Steel Plate, Sheet, and Strip for Pressure Vessels and for General Applications, ASTM International, West Conshohocken, PA, 2017.

[21] HO, K. H., NEWMAN, S. T., "State of the art electrical discharge machining (EDM)", International Journal of Machine Tools and Manufacture, v. 43, n. 13, pp. 1287-1300, 2003.

[22] MORAIS, N.W.S., VIANA, N.F., ABREU, H.F.G., "Comparação entre as técnicas de segmentação de imagens, difração de raios x e ferritoscopia na quantificação da martensita induzida por deformação no aço AISI 301L", Matéria (Rio J.), Rio de Janeiro. v. 16, n. 4, pp. 836-841, 2011.

[23] Operators Manual Feritscope ${ }^{\circledR}$ FMP30, Institutfür Elektronik und Messtechnik, Sindelfingen Germany. 2008. https://archive-resources.coleparmer.com/Manual_pdfs/59971-17,\%20-18.pdf

[24] CHEN, X., ZHOU, C., ZHENG, J., et al., "Effects of $\alpha^{\prime}$ martensite and deformation twin on hydrogenassisted fatigue crack growth in cold/warm-rolled type 304 stainless steel", International Journal of Hydrogen Energy, v. 43, n. 6, p. 3342-3352, 2018.

[25] FAHR, D., "Stress-and strain-induced formation of martensite and its effects on strength and ductility of metastable austenitic stainless steels", Metallurgical Transactions, v. 2, n. 7, pp. 1883-1892, 1971.

[26] ASTM E92-17, Standard Test Methods for Vickers Hardness and Knoop Hardness of Metallic Materials, ASTM International, West Conshohocken, PA, 2017, www.astm.org

[27] MARESCA, F., KOUZNETSOVA, V. G., GEERS, M. G. D., et al., "Contribution of austenitemartensite transformation to deformability of advanced high strength steels: from atomistic mechanisms to microstructural response", Acta Materialia, pp.463-478, 2018.

[28] BHADESHIA, H. K. D. H., WAYMAN, C. M., "Phase Transformations: Nondiffusive", In: Physical Metallurgy (Fifth Edition), pp. 1021-1072, 2015.

[29] HUANG, C. X., YANG, G., GAO, Y. L. et al., "Investigation on the nucleation mechanism of deformation-induced martensite in an austenitic stainless steel under severe plastic deformation", Journal of Materials Research, v. 22, n. 3, pp. 724-729, 2007.

[30] BELYAKOV, A., ODNOBOKOVA, M., KIPELOVA, A. et al., "Microstructural Evolution and Strengthening of Stainless Steels During Cold Rolling”, In: Frontiers in Materials Processing, Applications, Research and Technology, Springer, Singapore, pp. 341-347, 2018.

[31] TANHAEI, S., GHEISARI, K., ZAREE, S. A., "Effect of cold rolling on the microstructural, magnetic, mechanical, and corrosion properties of AISI 316L austenitic stainless steel", International Journal of Minerals, Metallurgy, and Materials, v. 25, n. 6, pp. 630-640, 2018.

[32] KRAUSS, G., "Martensite in steel: strength and structure", Materials Science and Engineering: A, v. 273, pp. 40-57, 1999.

[33] OGAWA, T., KOYAMA, M., TASAN et al., "Effects of martensitic transformability and dynamic strain age hardenability on plasticity in metastable austenitic steels containing carbon", Journal of Materials Science, v. 52, n. 13, pp. 7868-7882, 2017.

[34] LICHTENFELD, J. A., VAN TYNE, C. J., MATAYA, M. C., "Effect of strain rate on stress-strain behavior of alloy 309 and 304L austenitic stainless steel", Metallurgical and Materials Transactions A, v. 37, n. 1, pp. 147-161, 2006.

[35] TALONEN, J., HÄNNINEN, H., "Damping properties of austenitic stainless steels containing straininduced martensite", Metallurgical and Materials Transactions A, v. 35, n. 8, pp. 2401-2406, 2004.

[36] CHANG, L., BURKE, M. G., SCENINI, F., "Stress corrosion crack initiation in machined type 316L austenitic stainless steel in simulated pressurized water reactor primary water", Corrosion Science, v. 138, pp. 
$54-65,2018$.

\section{ORCID}

Allan Victor Mathias Marques

https://orcid.org/0000-0001-8377-2986

Kerciely Martins do Carmo https://orcid.org/0000-0002-0577-4975

Wivyan Castro Lage https://orcid.org/0000-0002-8435-8119

Ricardo Luiz Perez Teixeira https://orcid.org/0000-0003-2641-4036

José Carlos de Lacerda http://orcid.org/0000-0001-7753-1713

Cynthia Helena Soares Bouças Teixeira https://orcid.org/0000-0001-7436-6564

Ricardo Shitsuka

https://orcid.org/0000-0003-2630-1541 